\title{
QUANTUM ESTIMATES OF ALPHA EMITTER LIFE TIME
}

\author{
Budi Santoso \\ Center For Partnership in Nuclear Technology, \\ National Nuclear Energy Agency (BATAN) \\ Puspiptek, Serpong
}

\begin{abstract}
QUANTUM ESTIMATES OF ALPHA EMITTER LIFE TIME. Quantum estimates of several alpha radioactive life time have been made using the probability of quantum tunneling through the nuclear potential barrier. It is assumed that for a given nucleus with mass number $\mathrm{A}$ and isotopic number $\mathrm{Z}$, there exists an alpha particle moving freely back and forth in the nucleus with mass and isotopic numbers A -4 and Z-2. If the probability of penetrating the nuclear potential barrier is $T$, then after $\mathrm{N}$ times $(\mathrm{N}=1 / T)$ hitting the barrier an alpha particle is emitted. To obtain the elapsed time for emitting an alpha particle requires $\mathrm{N}$ times $\tau_{0}$, where $\tau_{0}$ is the time travel for alpha across the nuclear diameter, which is dependent on alpha energy. It is assumed here that this kinetic energy is the same as the emitted energy. The emitting alpha kinetic energies here are calculated by the difference of the masses of the parent and daughter nuclei and the alpha particles. They are in closed agreement with the experimental observations. While the alpha radioactive life time are not the same order of magnitudes but give the same linearity on the logarithmic scale as function of the inverse square root of energy.
\end{abstract}

\section{INTRODUCTION}

Alpha emitting nuclei have half life ranging from $10^{-7}$ seconds to something $10^{33}$ seconds. For instance the alpha emitting polonium ( $\mathrm{Po}_{84}^{212}$ ) has a half life of only $3 \times 10^{-7}$ seconds, while at the other extreme uranium isotope $\mathrm{U}_{92}^{238}$ has a half life of $4.5 \times 10^{9}$ years [1]. There is an enormous range encountered in the life time of alpha emitters. It has been found empirically the correlation between the life time of the nucleus and the emitted alpha particle energy. Typically the energies of the emitted alpha particles ranging from 4 to $10 \mathrm{MeV}$ [2].

The purpose of this study is to find the correlation function of the observed life time and the alpha energy, using a model of quantum tunneling within the nucleus. In this model, it is required to know the kinetic energy of alpha particle 'trapped ' in the nucleus.

For given alpha emitter nucleus with mass number $\mathrm{A}$ and isotopic number $\mathrm{Z}, \mathrm{m}(\mathrm{A}, \mathrm{Z})$ we can assume that the energetically emitted alpha particle has the kinetic energy equals to the mass difference of $m(A, Z)$ and $\mathrm{m}(\mathrm{A}-4, \mathrm{Z}-2)+\mathrm{m}(4,2)$ [3]. Data on nuclear mass have been tabulated in the nuclear data, meanwhile for practical purposes, we have here used the data 
listed by Elton [4]. Using this assumption, we got the energy estimates of the emitted alpha particles reasonably well fits the graph of Whichmann [2].

In this study the liquid drop model $[5,6,7]$ is also used to estimate the alpha emitted energy, but the error of the calculations are often greater than the precise values required. Hence, the values of nuclear mass are taken from the experimental data as for instance listed by Elton mentioned above.

\section{THEORETICAL BACKGROUND}

Quantum tunneling can be analyzed from the wave properties of particle. Consider a beam of alpha particle with energy $E$, traveling in a medium, in the form of a plane wave packet (represented by figure 1) incident on a potential wall, height $\mathrm{V}(\mathrm{x})$ and thickness $\mathrm{dx}$.

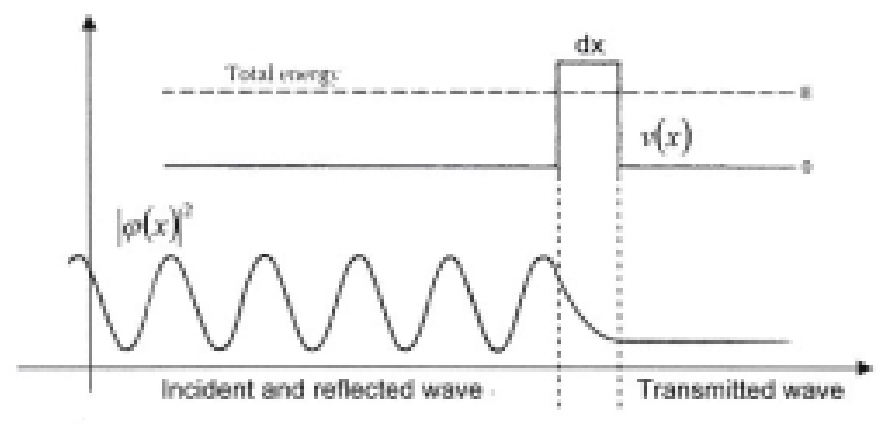

Region I II III

Figure 1. Incident of alpha particle on a square well potential barrier $\mathrm{V}(\mathrm{x})$ with the thickness dx.

Let divide the encounter into three regions, region I on the left is the region before encounter, region II, during the encounter and region III after encounter.

In each region it follows the wave equations:

Region I : $\quad \frac{d^{2}}{d x^{2}} \psi_{I}(x)+k^{2} \psi_{I}(x)=0$

Region II : $\frac{d^{2}}{d x^{2}} \psi_{I I}(x)-\kappa^{2} \psi_{I I}(x)=0$

Region III : $\frac{d^{2}}{d x^{2}} \psi_{I I I}(x)+k^{2} \psi_{I I I}(x)=0$ 
Where $k^{2}=\frac{2 m}{\hbar^{2}} E, \mathrm{~m}$ and $\mathrm{E}$ are being the mass and energy of alpha particle respectively, while $\kappa^{2}=\frac{2 m}{\hbar^{2}}\left(V_{0}-E\right)$. The solution of the wave equation in region $I$ is

$\psi_{I}(x)=e^{i k x}+A e^{-i k x}$

In region II

$\psi_{I I}(x)=B e^{-\kappa x}$

In region III

$\psi_{I I I}(x)=e^{i k x}$

Inserting the continuity conditions at the boundaries of the three regions, one get the coefficient $B \cong e^{-\kappa d x}$. This is the amplitude of the wave penetrating into region III. The probability of particle transmission is equal to the square of this magnitude. Therefore the probability of particle transmission is

$$
T=|B|^{2} \approx \exp (-2 \kappa d x)=\exp \left\{-2 d x \sqrt{\frac{2 m\left(V_{0}-E\right)}{\hbar^{2}}}\right\}
$$

If now $V_{0}$ is replaced by a Coulomb potential barrier in the form

$$
V(x)=\frac{2 e^{2} Z^{\prime}}{x}
$$

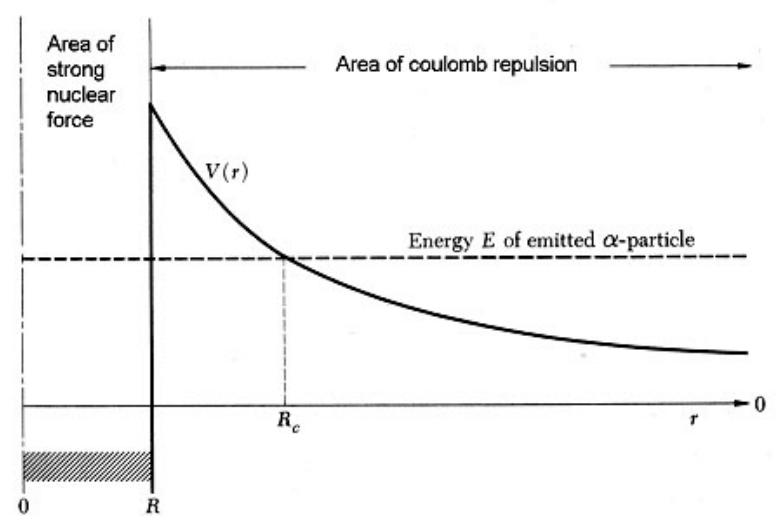

Figure 2. Alpha particle penetrating the coulomb potential barrier. 
One can divide the potential into smaller segment with thickness $d x$, each segment will give the transmission coefficient $T_{1}, T_{2}, T_{3} \ldots \ldots$, so that the probability of the transmission is equal to

$T=T_{1} T_{2} T_{3} \ldots$

Or

$\operatorname{Ln}(T)=\sum T_{i}=\int_{R}^{R_{c}}-2 d x \sqrt{\frac{2 m_{\alpha}\left(2 Z^{\prime} / x-E\right)}{\hbar^{2}}}$

The integral can be performed remembering that

$$
\begin{aligned}
& R=r_{0} A^{1 / 3}, \\
& R_{c}=\frac{2 e^{2} Z^{\prime}}{E}, \\
& Z^{\prime}=Z-2 \\
& V(x)=2 Z^{\prime} e^{2} / x \\
& \operatorname{Ln}(T) \cong-\frac{2 m \pi e^{2} Z^{\prime}}{\hbar} \sqrt{\frac{2 m_{\alpha}}{E}}+\frac{8}{\hbar} \sqrt{e^{2} Z^{\prime} R m_{\alpha}}
\end{aligned}
$$

Once $T$ is known, then after $\mathrm{N}=1 / T$, alpha particle will appear, and the time taken for the alpha particle to have $\mathrm{N}$ times hitting the potential wall is considered to be the life time. (In this case the half life time).

Whichman [2] has used the average values for $Z^{\prime}$ to be $Z^{\prime}=226, \quad R=7.3$ fermi, which are the values of these parameters for the case when the radium isotope $R a_{88}^{226}$ is the parent. Using these parameters he obtained

$$
\log (T / \mathrm{sec}) \cong-\frac{148}{\sqrt{E / M e V}}+32.5
$$

The time taken to oscillate back and forth in one shot within the nuclear potential is simply

$$
\tau_{0}=\frac{2 R}{v}, \quad v=\sqrt{\frac{2 E}{m_{\alpha}}}
$$

Therefore the alpha radioactive lifetime is given by

$$
\tau=N \tau_{0}=\frac{\tau_{0}}{T}
$$




$$
\log (\tau / \mathrm{sec})=\operatorname{Ln}\left(\tau_{0}\right)+\frac{148}{\sqrt{E / M e V}}-32.5
$$

Again using the Radium isotope parameters mentioned above, and using a "standard" alpha emitter, $\tau_{0} \cong 10^{-21} \mathrm{sec}$, one can come to a simple relation between the life time and the alpha kinetic energy

$$
\log (\tau / \mathrm{sec})=\frac{148}{\sqrt{E / M e V}}-53.5
$$

From this last expression, one can see the linear relation between the logarithmic of the life time ( in second) versus the inverse square root of alpha particle energy (in $\mathrm{MeV}$ ).

\section{CALCULATIONS OF ALPHA ENERGIES}

In the process of spontaneous alpha decay, the parent nucleus loses two protons and two neutrons, so that its mass decreases by four units and its charge by two units i.e. $\Delta A=-4, \quad \Delta Z=-2$. The disintegration can be represented by

$$
X_{Z}^{A}=Y_{Z-2}^{A-4}+H e_{2}^{4}
$$

The kinetic energy of the alpha particle (neglecting the impact on the parent nucleus) is given by

$$
Q=[M(A, Z)-M(A-4, Z-2)-m(4,2)] c^{2}
$$

The values of masses can be calculated from the formula derived from the liquid drop model [4]

$$
\begin{aligned}
& m(A, Z)=0 . .9917527 A-0.000839 Z+0.019114 A^{1 / 3}+0.0007626 \frac{Z^{2}}{A^{111 / 3}} \\
& +0.10175 \frac{[(A / 2)-Z]^{2}}{A}+\delta(A, Z)
\end{aligned}
$$

where the value of $\delta(A, Z)$ is given by

$$
\delta(A, Z)=\begin{array}{cccc}
-0.036 A^{-3 / 4} \text { for } & \text { even }-A, \text { even } & Z \\
0 & \text { for } & \text { odd } A & \\
& +0.036 A^{-3 / 4} \text { for } & \text { even }-A, \text { odd } & Z
\end{array}
$$


However the accuracy of this formula come to two significant digital numbers in milli mass unit, while the values of masses obtained from the experimental data can go to micro mass unit, as required in the calculations.

\section{RESULTS AND DISCUSSIONS}

In table 1 we have tabulated the values of nuclides that go alpha disintegration, indicated by its mass and atomic numbers, followed by their alpha energies. The calculation of alpha energy is compared to the observed values available from the data[6]. The formula given by (15) is actually a simplified where the variation in A and Z' are neglected. This is true because, the alpha emitters are all heavy nuclei, and the variation in A and $Z$ ' is small. Nevertheless, the variation I A and Z' can be included, and it is found the correction terms do not give significant improvement. Some results are numerically of the same order to the experimental values. Further study is being made as to what physical parameters should be introduced to improve the solutions.

In fig 3, we present the theoretical plot compared to the data as also shown by Whichmann [2].

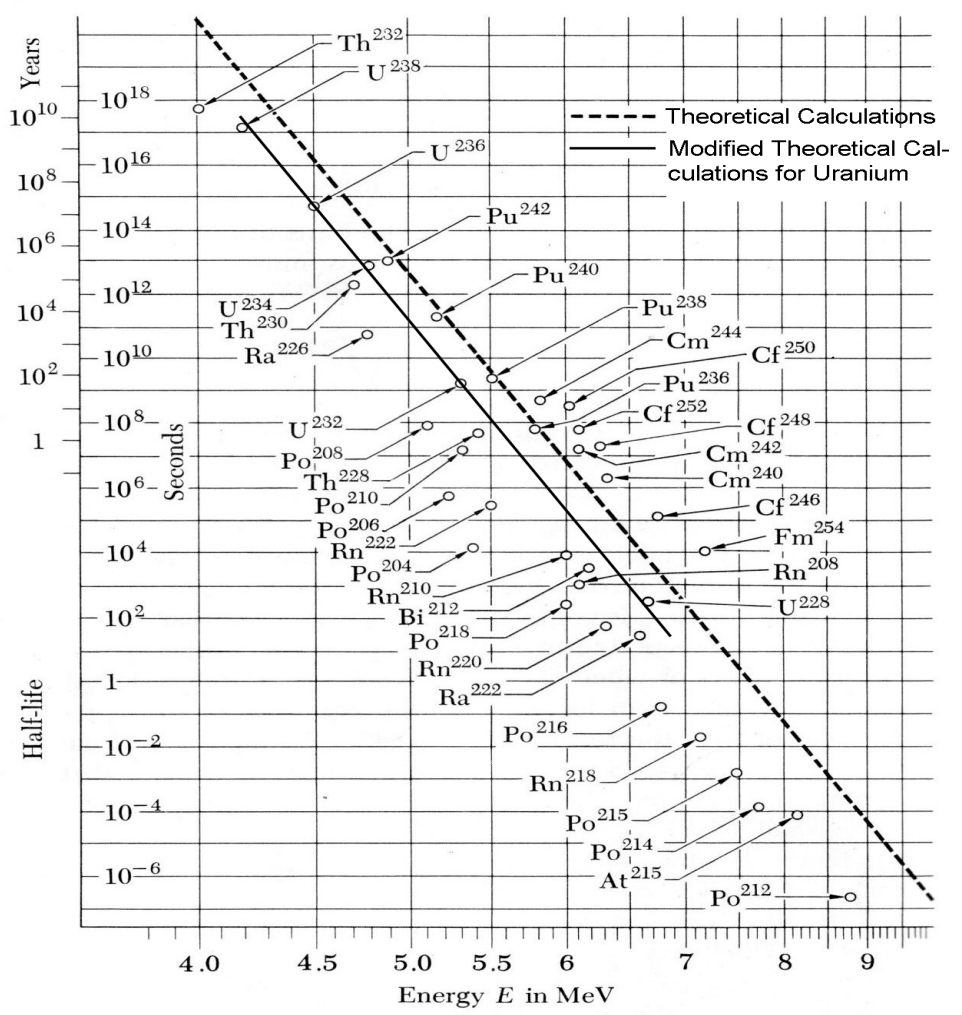

Figure 3. Half life time of alpha emitters versus energy. 
As can be seen from this plot, the circles are the actual observed data [10]. The theory predicts correlation of the logarithm of the half life (as the ordinate) and the quantity $-1 / \sqrt{E}$ abscissa, where $\mathrm{E}$ is the kinetic energy of the emitted alpha particle. These simple quantum estimates do not give good agreement with the experimental values, however the trend of dependence of half life on energy is correctly demonstrated. For the case of plutonium, the plot of theoretical calculations is relatively well fitted with the data values.

In the case of alpha decay for a number of uranium as parent nuclei, it is found empirically, that the half life versus energy, fits relatively well the observed data using an empirical plot given by

$$
\log (\tau / \mathrm{sec})=\frac{148}{\sqrt{E / M e V}}-55.0
$$

The last term in this expression contains the values of $Z^{\prime}$ and $R$ ( the isotope number and radius of parent nucleus) experienced by the alpha particle as an average values, which are not the same as the model considered.

\section{CONCLUSIONS}

We conclude that the quantum estimates give the correct trend of plot for the correlation between the half life and the energy of emitted alpha particle. In particular for the 'plutonium series', the theoretical plot is relatively well fit the data, while for the 'uranium series', a modified version is required to fit the data. This sheds a considerable light on the average potential experienced by the alpha particle inside the nucleus may different from the model to be considered. In the case of energy estimates, the calculation is based on energy conservation neglecting impact of the parent nucleus, giving relatively close agreement with the data available.

Table 1. Comparison of calculated and data of alpha energy.

\begin{tabular}{|c|c|c|c|}
\hline $\boldsymbol{A}$ & $\boldsymbol{Z}$ & $\boldsymbol{E}$ data & $\boldsymbol{E}$ calculated \\
\hline & & & Cf(Californium) \\
\hline 252 & 98 & 6.1 & 6.23 \\
248 & 98 & 6.25 & 6.37 \\
246 & 98 & & 6.87 \\
250 & 98 & 6.26 & 6.13 \\
\hline & & & Cm(Curium) \\
\hline 240 & 96 & & 6.40 \\
244 & 96 & 5.8 & 5.90 \\
242 & 96 & 6.1 & 6.21 \\
\hline
\end{tabular}




\begin{tabular}{|c|c|c|c|}
\hline & & & Pu(Plutonium) \\
\hline 240 & 94 & 5.25 & 5.25 \\
238 & 94 & & 5.60 \\
236 & 94 & 5.8 & 5.86 \\
242 & 94 & 4.9 & 4.98 \\
\hline & & & U(Uranium) \\
\hline 236 & 92 & & 4.58 \\
238 & 92 & & 4.26 \\
232 & 92 & 5.3 & 5.42 \\
234 & 92 & 4.8 & 4.83 \\
228 & 92 & & 6.79 \\
\hline & & & Th(Thorium) \\
\hline 230 & 90 & 4.7 & 4.78 \\
232 & 90 & & 4.07 \\
228 & 90 & 5.4 & 5.52 \\
\hline & & & Ra(Radium) \\
\hline 226 & 88 & 4.7 & 4.86 \\
222 & 88 & & 6.68 \\
208 & 86 & & 6.88 \\
222 & 86 & & 5.59 \\
220 & 86 & & 6.40 \\
210 & 86 & & 6.15 \\
218 & 86 & & 7.25 \\
\hline & & & Po(Polonium) \\
\hline 218 & 84 & & 6.10 \\
210 & 84 & 5.75 & 5.42 \\
216 & 84 & & 6.89 \\
208 & 84 & 5.1 & 5.20 \\
215 & 84 & & 7.51 \\
214 & 84 & & 7.83 \\
204 & 84 & & 8.95 \\
212 & 84 & & 6.20 \\
212 & 83 & & \\
& & & \\
\hline
\end{tabular}

\section{REFERENCES}

1. BOHR N, Quantized Energy Levels, Phil Mag, 26, 1, (1913).

2. SEGRE E., Experimental Nuclear Physics, John Wiley \& Sons Inc, USA, (1953).

3. ELTON LRB, "Introductory Nuclear Theory" ,The University Press Aberdeen Press, (1965). 
4. ARYA, P.ATAM, "Fundamentals of Nuclear Physics", Allyn and Bacon, Inc, Boston, (1966).

5. WHICHMANN, H. EYVIND, Quantum Physics, McGraw-Hill, San Francisco, (1971).

6. KAPLAN IRVING, Nuclear Physics, Addison-Wesley Publ Co, (1975).

7. GODFREY STEPHEN, "Liquid Drop Model", Internet Communication, (1997).

8. VINCENT STUART, "Liquid Drop Model", Internet Communication, (1999).

9. KEENAM ALEX, "Liquid Drop Model", Internet Communication, (2000).

10. HORIGUCHI T, TACHIBANA T, TAMURA $\mathrm{T}$, "Cart of The Nuclides", Tokaimura, Ibaraki, Japan, (1992). 